\title{
Spatial mode switchable, wavelength tunable erbium doped fiber laser incorporating a spatial light modulator
}

\author{
Y. Jung, ' Z. Li, N.H.-L. Wong, J. M. O. Daniel, J. K. Sahu, S.U. Alam, and D.J. Richardson \\ Optoelectronics Research Centre, University of Southampton, Southampton, SO17 1BJ, UK \\ *ymj@orc.soton.ac.uk
}

\begin{abstract}
We present a 2-mode group, switchable spatial mode erbium doped fiber laser incorporating a spatial light modulator. The laser wavelength can be tuned using an intra-cavity wavelength selective filter and provides $>10 \mathrm{~dB}$ extinction ratio between $\mathrm{LP}_{01} / \mathrm{LP}_{11}$ modes. OCIS codes: (060.3510) Lasers, fiber; (060.2410) Fibers, erbium; (060.0060) Fiber optics and optical communications.
\end{abstract}

\section{Introduction}

Fiber lasers [1] offer excellent beam quality, reliability, compactness and high photon conversion efficiency and have been widely used in diverse fields including optical communications, medicine, spectroscopy and material processing. Typically, fiber lasers are designed to operate on the fundamental spatial mode and the high beam quality enables applications such as fine laser marking. Multimode fiber lasers by contrast are typically used to generate the higher output powers needed for many industrial applications such as cutting, welding and heat treatment of metals. Recently, few-mode fibers have been used for mode division multiplexed (MDM) data transmission [2,3] where information is simultaneously transmitted on several spatial modes within a single optical fiber, and inline few-mode fiber amplifiers have been used to boost the transmission. In MDM optical networks selective excitation and detection of light propagating on the different transverse modes is a critical issue and proper modal characterization of the subsystems and fiber components needed is a key requirement. To this end a transverse mode switchable and wavelength tunable fiber laser offering well defined modal output at relatively high output powers would represent a very useful tool. In ref. [4], a mode selective fiber laser based on a multimode fiber Bragg grating (FBG) has been introduced. However, the operating wavelengths of the lowest two transverse modes $\left(\mathrm{LP}_{01}\right.$ and $\left.\mathrm{LP}_{11}\right)$ is achieved at different wavelengths with $\sim 5 \mathrm{~nm}$ separation due to the very different Bragg resonance conditions for each mode, while the wavelength tuning range of the fiber laser is fixed (or is necessarily narrow) due to the relatively limited tuning range associated with fiber Bragg gratings. In a recent publication in Nat. Comm. [5], a reflective phase-only spatial light modulator (SLM) was used as an intra-cavity holographic mirror in a standard (bulk crystal) solid-state laser resonator and showed the feasibility of electronically controlling the spatial transverse mode of the laser. This represents the first "mode on demand" operation of a solid-state laser and the high resolution of the SLM enables precise and highly flexible intra-cavity laser beam shaping.

In this paper, we employ such a high-speed, electronically addressable SLM device as a cavity mirror in an erbium doped fiber laser cavity and demonstrate digital electronic control of the spatial output mode. In this case selection of the transverse spatial mode requires only a change to the phase pattern applied to the SLM and no additional alignment to the fiber laser cavity is required. Furthermore, a tunable bandpass filter is incorporated within the cavity to allow wavelength tuning. We successfully demonstrate selective lasing of either the fundamental $\left(\mathrm{LP}_{01}\right)$, or next high order mode $\left(\mathrm{LP}_{11}\right)$ in a core-pumped erbium doped fiber laser supporting two mode groups and show wavelength tuning over a large fraction of the C-band (limited only by the long wavelength tuning of the filter).

\section{Experimental setup}

The experimental setup for the proposed transverse mode switchable and wavelength tunable fiber laser is illustrated in Fig. 1(a). A two mode group erbium doped fiber (EDF) was used as the active gain medium and a short length $(\sim 10 \mathrm{~m})$ of passive two mode fiber (TMF) was spliced at both ends of the active fiber to reduce the alignment complexity (if needs these passive fibers could ultimately be removed). Both end facets of the fiber were end-capped with a coreless silica fiber (diameter $=200 \mu \mathrm{m}$, length $=300 \mu \mathrm{m}$ ) to suppress broadband feedback and to reduce potential for parasitic lasing. In our experiment, a 5m long ring-doped EDF was used, in which the erbium ions are substantially confined within a ring inside the fiber core to help mitigate the differential modal gain as needed for MDM transmission [6]. The TM-EDF has an outer cladding diameter of $111 \mu \mathrm{m}$ and an inner core diameter of $7.7 \mu \mathrm{m}$. The estimated effective NA of the core is $\sim 0.14$. The measured absorption at $980 \mathrm{~nm}$ is $5.56 \mathrm{~dB} / \mathrm{m}$ and the background loss is $21.4 \mathrm{~dB} / \mathrm{km}$ at $1285 \mathrm{~nm}$. The laser cavity was defined by a silver mirror (100\% reflectivity) at one end of the fiber with a spatial light modulator based reconfigurable digital mirror at the other. The SLM used in our experiment is a reflective phase-only liquid crystal display (Holoeye Pluto-Telco, 1920x1080 pixel resolution, $8 \mu \mathrm{m}$ pixel pitch) optimized for $1550 \mathrm{~nm}$. The linear polarization axis of the incoming beam (defined by an intracavity 
polarizer) was aligned to match the orientation of the liquid crystals in the SLM in order to ensure the highest possible phase modulation efficiency.

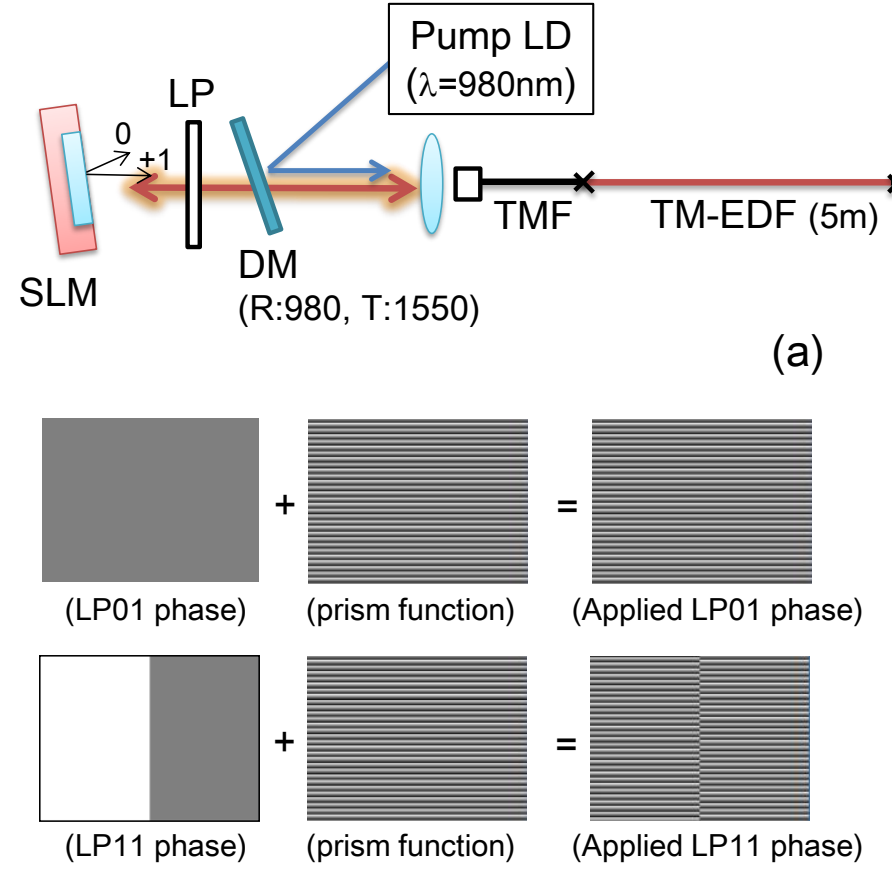

(b)
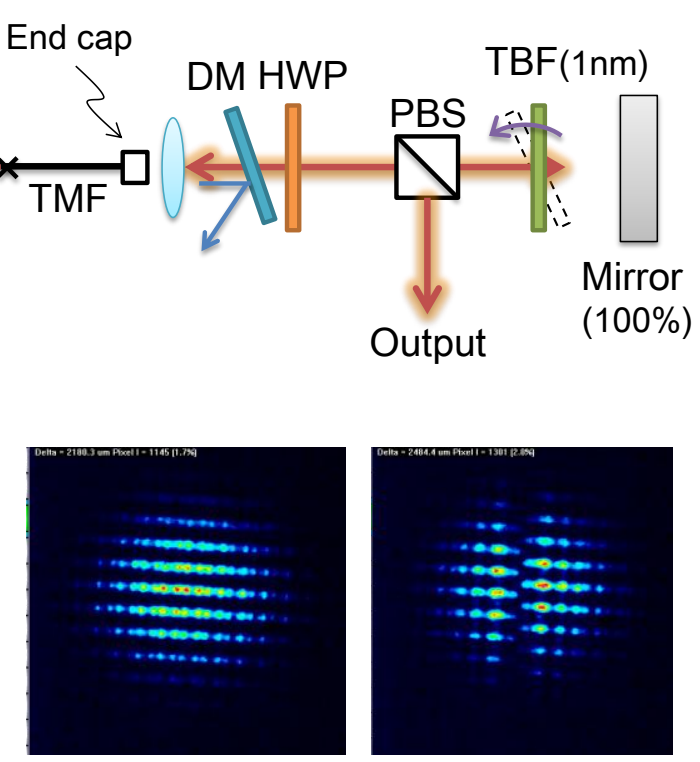

(LP01 diffracted beam) (LP11 diffracted beam)

(c)

Fig. 1. (a) Schematic of transverse mode switchable and wavelength tunable fiber laser employing a SLM device for intra-cavity beam shaping, (b) applied phase patterns to generate $\mathrm{LP}_{01}$ and $\mathrm{LP}_{11}$ modes and (c) CCD images of the diffracted light from the SLM panel

A 980nm single mode pump laser was free-space coupled into the TM-EDF following reflection from a dichroic mirror (high reflection @980nm and high transmission@1550nm). The half wave plate (HWP) at the other end of the EDF controls the laser output coupling at the polarization beam splitter (PBS). The PBS acts both as the output coupler and to provide polarization-dependent loss. A narrow bandwidth $(\Delta \lambda=1 \mathrm{~nm})$ optical tunable bandpass filter $(\mathrm{TBF})$ is used within the cavity to select the lasing wavelength. In our experiment, the binary phase pattern from the SLM produces a relative phase delay for each pixel ranging from 0 to $2 \pi$ and can be used to generate $\mathrm{LP}_{01}$ and $\mathrm{LP}_{11^{-}}$ like beams. To enhance the modal purity and to separate the controlled diffracted beam from the reflected beam, we superimposed a prism phase in the $\mathrm{Y}$ direction on the displayed image which provides the optical phase function of a prism in the $\mathrm{Y}$ direction and splits the diffracted beam from the reflected beam. Therefore, the final phase pattern applied to the SLM corresponds to a combination of a guided fiber mode and grating phase, as shown in Fig. 1(b). CCD images of the diffracted light from the SLM panel are shown in Fig. 1(c).

(a)

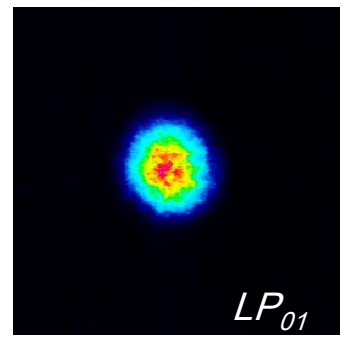

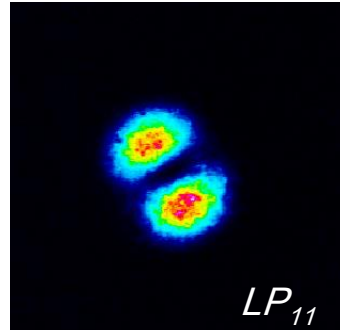

(b)

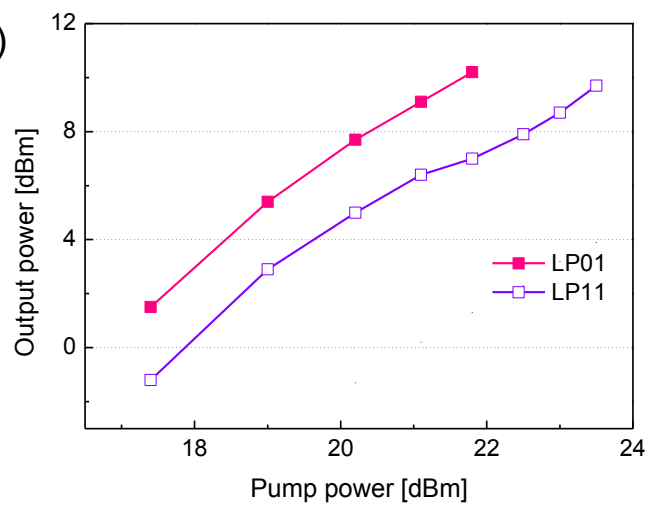

Fig. 2. (a) Measured transverse mode profiles and (b) laser output power as a function of pump power for two different transverse modes. 


\section{Fiber laser performance and characterizations}

The SLM performs the function of a selective mode excitation/blocker which favors the transmission of a particular mode, but which largely suppresses other transverse modes. The measured output beam profiles after the PBS are shown in Fig. 2(a). As of yet we have not quantitatively analyzed the mode purity using a few mode fiber demultiplexing setup, but quite clean mode profiles were observed with a CCD camera and we estimate an ER of at least $10 \mathrm{~dB}$. When the laser oscillates at $1550 \mathrm{~nm}$, the lasing output power as a function of pump power for both transverse modes was investigated. As shown in Fig. 2(b), the laser output power of both transverse modes increases linearly with launched pump power at powers above the (mode-dependent) laser threshold. At 150mW of launched pump power the laser yielded $10.5 \mathrm{~mW}$ of output power for the $\mathrm{LP}_{01}$ mode and $5.0 \mathrm{~mW}$ for the $\mathrm{LP}_{11}$ mode, correspond to overall laser efficiencies of $9 \%$ and $4 \%$ respectively. The higher threshold for LP $\mathrm{P}_{11}$ mode operation is attributed to the higher cavity loss and the lesser spatial overlap of the signal and pump modes (in this current experiment a simple central pump launch scheme was used, resulting in the fundamental mode experiencing higher gain compared to the high order mode [6]). The relatively low damage threshold of the SLM $\left(\sim 2 \mathrm{~W} / \mathrm{cm}^{2}\right)$ currently limits the maximum output power to $10 \mathrm{~mW}$ but we believe that the output power could be substantially further increased by optimizing the free space components and more careful position of the SLM relative to the output coupler within the cavity.
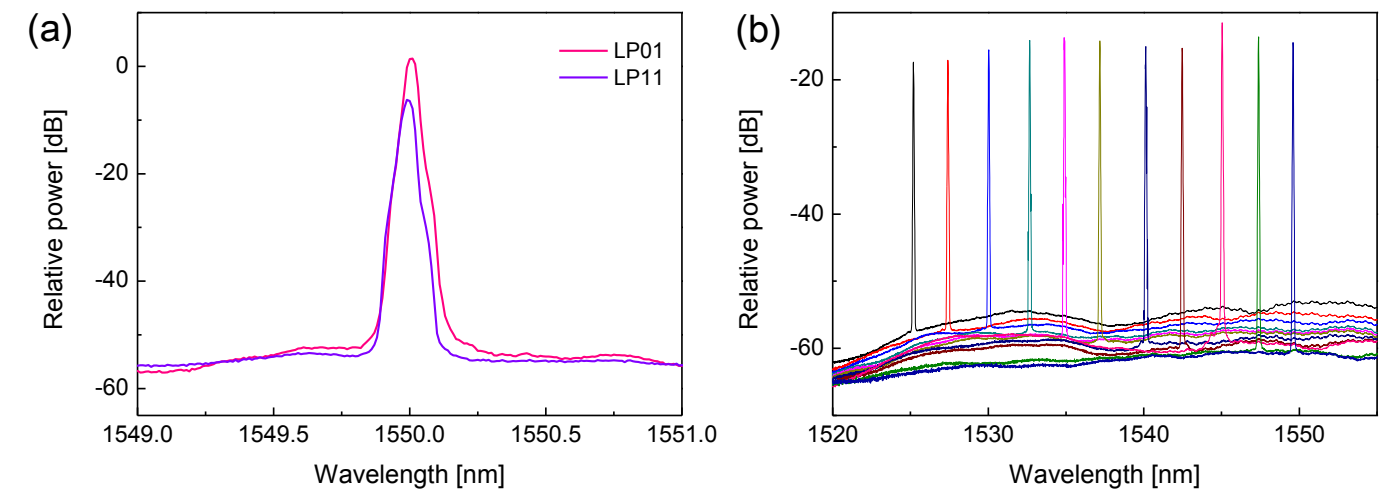

Fig. 3. (a) Measured output spectra of two transverse modes and (b) wavelength tuning over C-band.

Fig. 3(a) depicts the optical spectra of the two transverse modes, measured using an ANDO optical spectrum analyzer (AQ6370B) with a 0.02-nm resolution, showing that there is negligible frequency shift on switching spatial mode. The $3 \mathrm{~dB}$ bandwidth is measured to be less than $0.04 \mathrm{~nm}$ while the signal-to-noise ratio is more than $50 \mathrm{~dB}$ for both $\mathrm{LP}_{01}$ and $\mathrm{LP}_{11}$ modes. By changing the tilt angle of the TBF it is possible to tune the lasing wavelength over the C-band. As shown in Fig. 3(b), the maximum wavelength of operation was limited to 1550nm due to the limited operating range of the tunable filter used in our experiment but we are confident that the laser could be made to operate over the entire C-band with an appropriate choice of filter.

\section{Conclusion}

We have successfully demonstrated a spatial mode switchable, wavelength tunable erbium doped fiber laser. A programmable reflective phase-only SLM was employed as an intra-cavity phase control element and clean LP 01 and $\mathrm{LP}_{11}$ modes were obtained from the erbium doped fiber laser supporting two mode groups. Maximum output powers of $10.5 \mathrm{~mW}$ and $5.0 \mathrm{~mW}$ were obtained for the $\mathrm{LP}_{01}$ and $\mathrm{LP}_{11}$ modes respectively. Further increases in output power will be possible by optimizing the intra-cavity losses. We believe that this unique spatial mode switchable and wavelength tunable fiber laser will prove a useful tool for testing the components and subsystems in future mode division multiplexing transmission systems.

This work was supported by the European Communities $7^{\text {th }}$ Framework Programme under grant agreement 258033 (MODE-GAP).

\section{References}

[1] D. J. Richardson, et. al., “High power fiber lasers: current status and future perspective,” J. Opt. Soc. Am. B 27, 63092 (2010).

[2] D. J. Richardson, et. al., "Space-division multiplexing in optical fibres," Nature Photonics 7, 354-362 (2013).

[3] V.A.J.M.Sleiffer, et. al., "73.7Tb/s (96x3x256-Gb/s) mode-division-multiplexed DP-16QAM transmission with inline MM-EDFA,” Opt. Express 20, B428-438 (2012).

[4] J.M.O.Daniel, et. al., "Novel technique for mode selection in a multimode fiber laser," Opt. Express 19, 12434-12439 (2011).

[5] S. Ngcobo, et. al., "A digital laser for on-demand laser modes," Nat. Comm. 4, 2289 (2013).

[6] Y. Jung, et. al., "Three mode Er3+ ring-doped fiber amplifier for mode-division multiplexed transmission," Opt. Express 21, 10383-10392 (2013). 